\title{
Nanoencapsulation of blocked isocyanates through aqueous emulsion polymerization
}

\author{
H. Yang, S. K. Mendon, J. W. Rawlins*
}

School of Polymers and High Performance Materials, The University of Southern Mississippi, 118 College Drive \#10037, Hattiesburg, MS 39406, USA

Received 14 February 2008; accepted in revised form 24 March 2008

\begin{abstract}
Blocked isocyanates were successfully encapsulated into polystyrene and hydroxyl and amine functionalized polymeric nanospheres via emulsion polymerization. The nanocapsules were characterized via Fourier transform infrared spectroscopy, differential scanning calorimetry and transmission electronic microscopy. The blocked isocyanates generated free isocyanate functionality upon thermal annealing of nanocapsules. This research establishes a novel encapsulating method for release and retention of free isocyanates in aqueous media. These nanocapsules can provide active isocyanates in coatings and adhesive applications, and represent a novel application of nanoencapsulated materials for controlled or delayed active material utilization.
\end{abstract}

Keywords: nanomaterials, nanoencapsulation, nanocapsules, emulsion polymerization, isocyanate

\section{Introduction}

The synthesis and application of nanoparticles, i.e., particles smaller than one micron, are of growing interest to both industry and academia. Nanocapsules, a specific class of nanoparticles, are composed of one or more active materials (core) and a protective matrix (shell) [1]. Encapsulation technology has been used to prepare micro/nanocapsules with specific application properties in several areas including food [2], biology [3], and medicine [4]. Nanocapsules have been synthesized via miniemulsion $[5,6]$ and interfacial polymerization $[7,8]$ techniques. Couvreur reviewed nanocapsule technologies used in drug carrier systems [9].

Most encapsulation techniques employ isocyanates in either solvent or bulk to form shell (or matrix) materials for encapsulating functional materials, releasable fill materials [10], or making pressure sensitive copying paper [11]. The inherent reactivity of isocyanates with water makes it difficult to encapsulate them in aqueous media. It is even more challenging to maintain isocyanate activity in the presence of hydroxyl- or amine-functional systems. There are no references found in literature for either the incorporation of blocked isocyanates into polymeric nanoparticles or the encapsulation of isocyanates into reactive polymeric shell materials in aqueous media.

In this study, blocked isocyanates were encapsulated within polystyrene nanocapsules and in hydroxyl and amine functionalized nanocapsules via emulsion polymerization. Upon thermal treatment, the blocking agent was released and active isocyanate functionalities were generated that remained stable in the aqueous medium within the protective shell of the nanocapsules. The nanocapsules were characterized via Fourier transform infrared (FTIR) spectroscopy, differential scanning calorimetry (DSC), and transmission electron microscopy (TEM). 


\section{Experimental section}

\subsection{Materials}

Desmodur $^{\circledR}$ N 3300A and Desmodur BL 3175A were received from Bayer MaterialScience, Pittsburgh, PA. 2-Hydroxyethyl methacrylate (HEMA), divinylbenzene (DVB), 2-(tert-butylamino)ethyl methacrylate (TBAEMA), 2,2'-azobisisobutyronitrile (AIBN), and sodium dodecyl sulfate (SDS) were purchased from Sigma-Aldrich, St. Louis, MO. Styrene was purchased from Alfa Aesar, Ward Hill, MA. Igepal ${ }^{\circledR}$ CO-887 was received from Rhodia, Cranbury, NJ. All reagents were used as received.

\subsection{Preparation of nanocapsules}

The nanocapsules were prepared through two steps of pre-emulsification (to form a pre-emulsion) and emulsion polymerization, which will be explained in detail below.

\subsubsection{Pre-emulsion preparation}

Pre-emulsion preparation for one of the nanocapsules (M-6) is provided as an example. The preemulsion was synthesized by blending two parts; Part I contained $40 \mathrm{~g}$ styrene, $0.8 \mathrm{~g}$ DVB, $0.82 \mathrm{~g}$ AIBN, and $40 \mathrm{~g}$ Desmodur BL3175A; and Part II contained $1.71 \mathrm{~g}$ SDS, $1.63 \mathrm{~g}$ Igepal CO-887, and $220 \mathrm{~g}$ water. Parts I and II were blended magnetically in separate containers for 10 minutes. Part II was then added to Part I under mechanical agitation and the contents were stirred for 30 minutes at $1800 \mathrm{rpm}$. The resulting pre-emulsion was cooled to $<5^{\circ} \mathrm{C}$ before sonication using a Misonix ${ }^{\circledR}$ sonicator 3000 (until a particle size $<250 \mathrm{~nm}$ was achieved).

\subsubsection{Emulsion polymerization}

The pre-emulsion was transferred to a three-neck round bottom flask equipped with a mechanical stirrer, reflux condenser, and a nitrogen inlet, and degassed for 30 minutes. The temperature was raised to $70^{\circ} \mathrm{C}$ and maintained for 8 hours to complete the polymerization.

\subsection{Characterization}

The latexes were freeze dried (lyophilization) for 48 hours in the Freezemobile 35EL. Glass transition temperatures $\left(T_{g} \mathrm{~s}\right)$ were recorded on a differential scanning calorimeter (DSC Q1000, TA Instruments, New Castle, DE), summarized in Table 1. FTIR spectroscopy (attenuated total reflectance and diffuse reflectance, unless specifically stated) was performed using a Scimitar series spectrophotometer (Digilab, Canton, MA). Latex particle sizes were recorded on a capillary hydrodynamic fractionator (CHDF 2000, Matec Applied Sciences, Northborough, MA). TEM was performed on a JEOL JEM-2100 microscope (JEOL Ltd., Tokyo, Japan).

For deblocking studies, the freeze dried samples were treated thermally in the DSC under a nitrogen atmosphere as summarized in Table 2 and characterized via FTIR.

For acetone extraction studies, the freeze dried samples were blended with acetone and agitated on a Vortex-Genie $2^{\mathrm{TM}}$ shaker for 48 hours. The blend was centrifuged using SPD SpeedVac ${ }^{\circledR}$ SPD 121P (Thermo Fisher Scientific, Waltham, MA), and the supernatant liquid was discarded (repeated twice, 20 minutes centrifugation). The pellets were air dried for further analysis.

Table 1. DSC Measurement procedure

\begin{tabular}{|l|c|c|c|}
\hline \multicolumn{1}{|c|}{ Step } & $\begin{array}{c}\text { Equilibrate } \\
{\left[{ }^{\circ} \mathbf{C}\right]}\end{array}$ & $\begin{array}{c}\text { Ramp to } \\
\left(\mathbf{a t ~ 1 0}{ }^{\circ} \mathbf{C} / \mathbf{m i n}\right) \\
{\left[{ }^{\circ} \mathbf{C}\right]}\end{array}$ & $\begin{array}{c}\text { Isothermal } \\
{[\mathbf{m i n}]}\end{array}$ \\
\hline I & -50 & - & - \\
\hline II (1 ${ }^{\text {st }}$ heating) & - & 120 & 3 \\
\hline III & - & -50 & 3 \\
\hline IV (2 ${ }^{\text {nd }}$ heating) & - & 180 & 3 \\
\hline V & - & -50 & 3 \\
\hline VI $\left(3^{\text {rd }}\right.$ heating $)$ & - & 180 & 3 \\
\hline VII & - & -50 & 3 \\
\hline VIII (4th heating) & - & 180 & - \\
\hline
\end{tabular}

Table 2. Deblocking treatment procedure

\begin{tabular}{|c|c|c|c|}
\hline Step & $\begin{array}{c}\text { Equilibrate } \\
{\left[{ }^{\circ} \mathbf{C}\right]}\end{array}$ & $\begin{array}{c}\text { Ramp to } \\
\left(\mathbf{a t} \mathbf{1 0}{ }^{\circ} \mathbf{C} / \mathbf{m i n}\right) \\
{\left[{ }^{\circ} \mathbf{C}\right]}\end{array}$ & $\begin{array}{c}\text { Isothermal } \\
{[\mathbf{m i n}]}\end{array}$ \\
\hline I & -50 & - & - \\
\hline II & - & 120 & 1 \\
\hline III & - & -50 & 3 \\
\hline IV & - & 160 & 40 \\
\hline V & - & -50 & 3 \\
\hline VI & - & 180 & - \\
\hline
\end{tabular}




\section{Results and discussion}

\subsection{Isocyanate encapsulated by polystyrene}

Blocked isocyanates (BNCO) are widely used in coatings, elastomers, composites, and rubber industries to thermally regenerate isocyanate functionalities. In this study, Desmodur BL3175A, a blocked aliphatic polyisocyanate based on hexamethylene diisocyanate $(75 \%$ solids, deblocking temperature $\sim 135^{\circ} \mathrm{C}$ ) was chosen for encapsulation into nanocapsules. The BNCO nanocapsules prepared via miniemulsion polymerization are summarized in Tables 3 and 4 (non-functionalized and functionalized nanocapsules are designated as $\mathrm{M}$ and $\mathrm{N}$ series, respectively). Desmodur N3300A (polymeric hexamethylene diisocyanate) was employed as the control for FTIR characterization. The latex particle sizes are included in the tables and were

Table 3. Blocked isocyanate (BNCO) nanocapsules

\begin{tabular}{|c|c|c|c|}
\hline Sample & DVB $^{*}$ [\%] & BNCO* [\%] & Particle size [nm] \\
\hline M-1 & 2 & 0 & 58 \\
\hline M-2 & 2 & 10 & 59 \\
\hline M-3 & 0 & 10 & 57 \\
\hline M-4 & 2 & 20 & 59 \\
\hline M-5 & 2 & 30 & 62 \\
\hline M-6 & 2 & 40 & 59 \\
\hline M-7 & 2 & 60 & 53 \\
\hline M-8 & 2 & 80 & 82 \\
\hline M-9 & 0 & 30 & 56 \\
\hline M-10 & 4 & 30 & 53 \\
\hline M-11 & 6 & 30 & 57 \\
\hline
\end{tabular}

*weight percentage on styrene

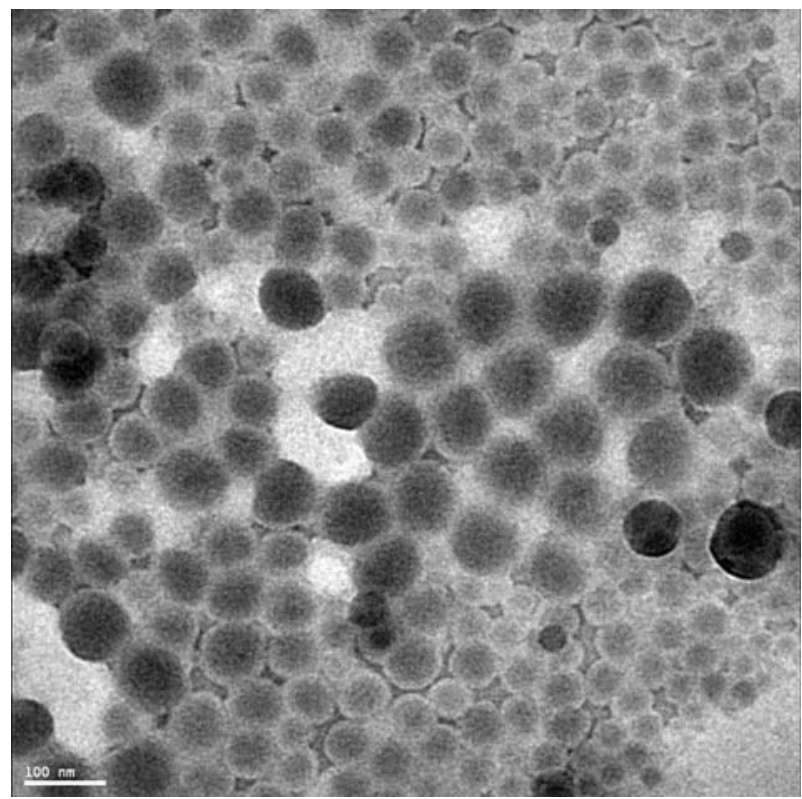

Figure 1. TEM photo of M-7 (scale bar is $100 \mathrm{~nm}$ ) also confirmed by TEM (TEM image of nanocapsule M-7 is shown as an example in Figure 1).

\subsubsection{FTIR analysis}

Isocyanates are characterized by peaks at 1682 (urethane $\mathrm{C}=\mathrm{O}$ stretching) and $2270 \mathrm{~cm}^{-1}(\mathrm{~N}=\mathrm{C}=\mathrm{O}$ stretching) for Desmodur N3300A, and at 1682 (urethane $\mathrm{C}=\mathrm{O}$ stretching) and $1725 \mathrm{~cm}^{-1}$ (blocked isocyanate $>\mathrm{C}=\mathrm{N}-\mathrm{O}-\mathrm{C}(\mathrm{O})-\mathrm{NH}, \mathrm{C}=\mathrm{O}$ stretching) for Desmodur BL3175A (Figures 2a and 2b). The presence of the characteristic BNCO peaks in the FTIR spectrum of the nanocapsule, M-6 (Figure 2e), and its absence in the FTIR spectrum of the hollow nanosphere, M-1 (Figure 2d), confirmed $\mathrm{BNCO}$ inclusion in the nanocapsules.

Nanocapsule M-6 was treated with acetone to extract the incorporated BNCO. The FTIR spectrum of M-6 after acetone extraction (Figure 2f) shows complete disappearance of the characteristic $\mathrm{BNCO}$ peaks indicating that the blocked isocyanate was extracted from the nanocapsules by acetone.

Nanocapsules containing the blocked isocyanates generated active isocyanates upon thermal treatment. For deblocking studies, the nanocapsules were freeze dried and treated thermally in the DSC to release the blocking agent. The FTIR spectrum of the BNCO nanocapsule M-6 subjected to deblocking treatment shows the characteristic isocyanate peak $\left(2270 \mathrm{~cm}^{-1}\right)$ (Figure $2 \mathrm{~g}$ ). Figure 2c displays the effect of the deblocking thermal treatment on the blocked isocyanate BL3175A. While

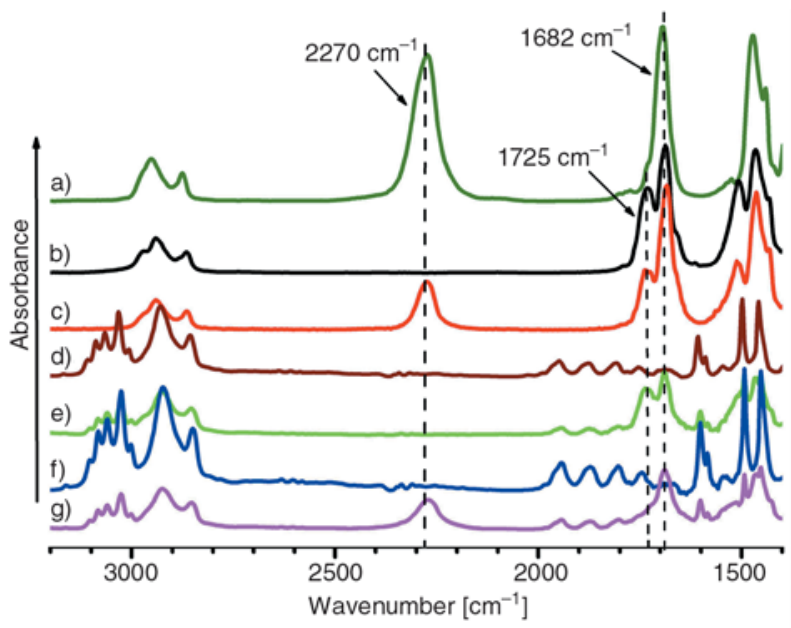

Figure 2. FTIR spectra of a) N3300A, b) BL3175A,

c) BL3175A after deblocking treatment, d) M-1,

e) M-6, f) M-6 after acetone extraction, and

g) M-6 after deblocking treatment 


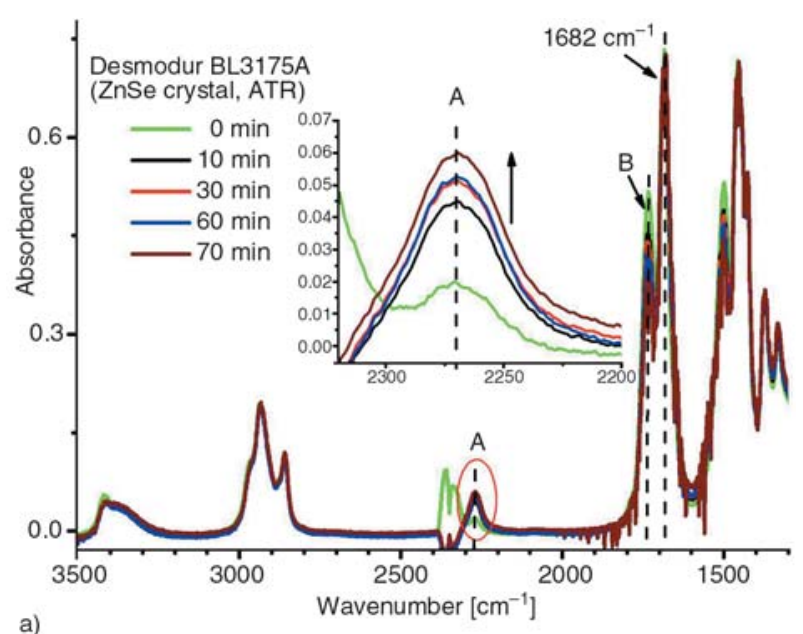

a)

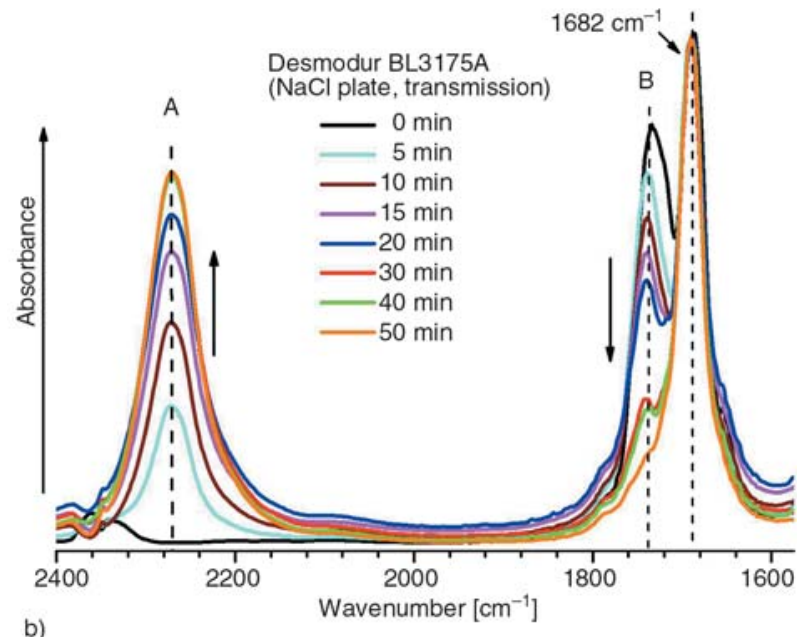

Figure 3. FTIR spectra of BL3175A maintained isothermally for various times at $140^{\circ} \mathrm{C}$ with a) ATR and b) transmission methods

the presence of the isocyanate peak at $2270 \mathrm{~cm}^{-1}$ indicates deblocking, the peak at $1725 \mathrm{~cm}^{-1}$ indicates that the BNCO was not completely deblocked by the thermal treatment. The deblocking appeared to be a function of time, therefore BL3175A and nanocapsule M-7 (2\% DVB, 60\% BNCO) were heated isothermally at $140^{\circ} \mathrm{C}$ for varying lengths of time and analyzed via FTIR (Figures 3 and 4).

In Figure 3a, the first curve for BL3175A (0 min, bottom line) reveals a small amount of deblocked $\mathrm{NCO}$, which indicates initiation of the deblocking process at $140^{\circ} \mathrm{C}$. Subsequent spectra show the effects of isothermal heating of BL3175A for varying lengths of time. The close-up profile (Figure 3a, area $\mathrm{A}$ ) shows increasing intensity of the $\mathrm{NCO}$ peak $\left(2270 \mathrm{~cm}^{-1}\right)$ with time. Even after 70 minutes at $140^{\circ} \mathrm{C}$ (top line), a small peak is visible at $1725 \mathrm{~cm}^{-1}$ (peak B) suggesting the presence of residual blocked isocyanate. Since the ATR method primarily probes only the top two microns of the

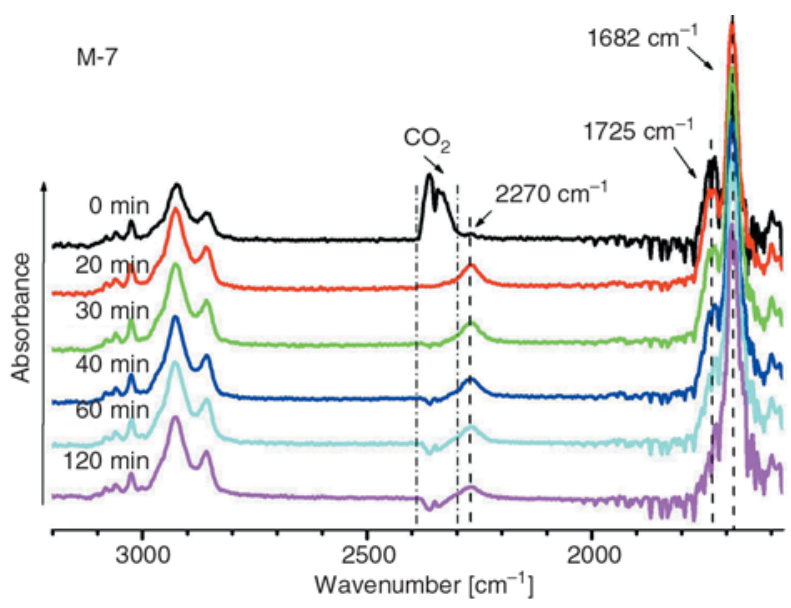

Figure 4. FTIR spectra of M-7 maintained isothermally for various times at $140^{\circ} \mathrm{C}$

sample that is in direct contact with the ZnSe crystal, the deblocking of BL3175A was also analyzed via transmission FTIR on sodium chloride plates (Figure $3 \mathrm{~b}$ ). To show the time-dependent deblocking property of $\mathrm{BNCO}$, the transmission FTIR data were re-plotted after normalization at $1682 \mathrm{~cm}^{-1}$. It is evident that the peak $A$ height increased while peak B decreased with time. Peak B disappeared after 50 minutes at $140^{\circ} \mathrm{C}$, suggesting that by this time, the isocyanate is completely dissociated via thermal treatment of blocked isocyanate.

A similar heating profile for the M-7 BNCO nanocapsule summarizes the effect of deblocking by the appearance and continued presence of deblocked isocyanate (Figure 4). At the end of 120 minutes, the $1725 \mathrm{~cm}^{-1}$ peak has almost disappeared indicating complete deblocking of the original $\mathrm{BNCO}$ and free NCO retention.

\subsubsection{Effects of crosslinking}

The blocking and deblocking of diisocyanates with methyl ethyl ketoxime is a reversible reaction [12]. In these nanocapsules, the polystyrene shell functions as a 'shield' for the deblocked isocyanate by preventing the liberated blocking agent from reentering the nanocapsule. As shown in Figure 5, varying the degree of crosslinking in the polystyrene shell further exemplifies the 'shield effect' (FTIR spectra of N3300A, BL3175A, and BL3175A after deblocking treatment are re-plotted to facilitate comparison).

After subjecting the nanocapsule M-9 (no DVB crosslinker in the shell) to the deblocking treatment, a small shoulder peak is revealed at 
$1725 \mathrm{~cm}^{-1}$, indicating the presence of residual BNCO (Figure 5f). Nanosphere M-1 (no blocked isocyanate) was blended with BL3175A in the same proportion as present in nanocapsule M-6 and subjected to deblocking treatment (Figure 5e). The

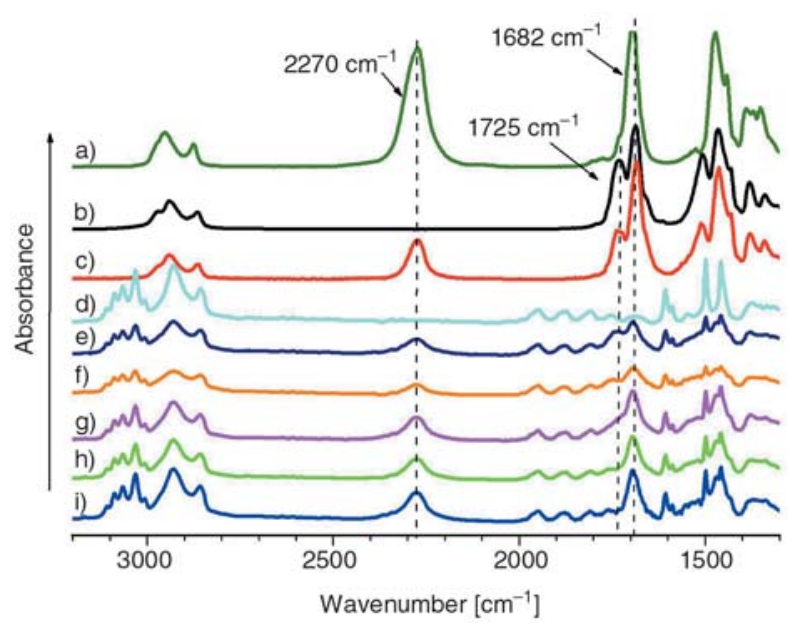

Figure 5. FTIR spectra of a) N 3300A, b) BL3175A,

c) BL3175A after deblocking treatment, d) M-1, e) M-1 and BL3175A blend after deblocking treatment, f) M-9 after deblocking treatment, g) M-5 after deblocking treatment, h) M-10 after deblocking treatment, and i) M-11 after deblocking treatment

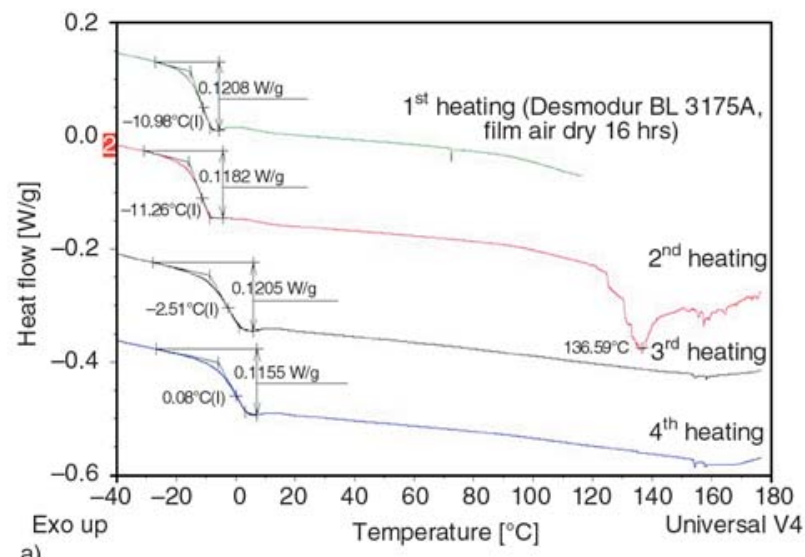

a)

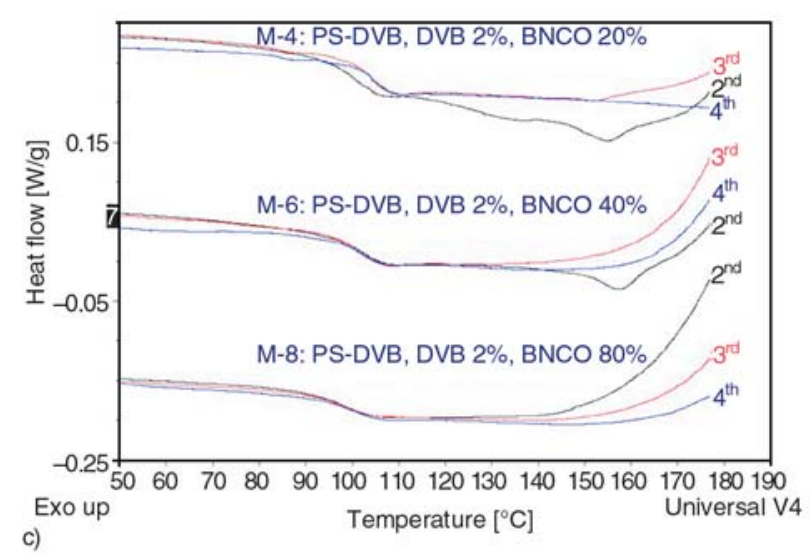

strong peak at $1725 \mathrm{~cm}^{-1}$ observed upon deblocking the blend indicates the effect of the polystyrene shell on the reaction between the isocyanate and the liberated blocking agent. Increasing the proportion of DVB from 2\% (M-5) to 6\% (M-11) results in shorter peaks at $1725 \mathrm{~cm}^{-1}$ (Figures $5 \mathrm{~h}$ and $5 \mathrm{i}$ ). The data validate that increasing the shell's degree of crosslinking (polystyrene shell) enhances the 'shield effect'.

\subsubsection{DSC analysis}

DSC thermograms of lyophilized nanocapsules are summarized in Figure 6. BL3175A (Figure 6a) exhibits a $T_{g}$ of $-11.26^{\circ} \mathrm{C}$ in the second heating cycle before the isocyanate deblocks at $136.59^{\circ} \mathrm{C}$. The $T_{g}$ shifted to $-2.51^{\circ} \mathrm{C}$ in the next heating cycle and the deblocking peaks were no longer evident. However, this does not signify complete deblocking of the blocked isocyanate. For instance, the DSC profile of the thermally treated BL3175A (Figure 2c), does not exhibit a deblocking thermal peak in the heating cycle following isothermal treatment at $160^{\circ} \mathrm{C}$ for 40 minutes but the IR spectrum of the same sample shows the presence of

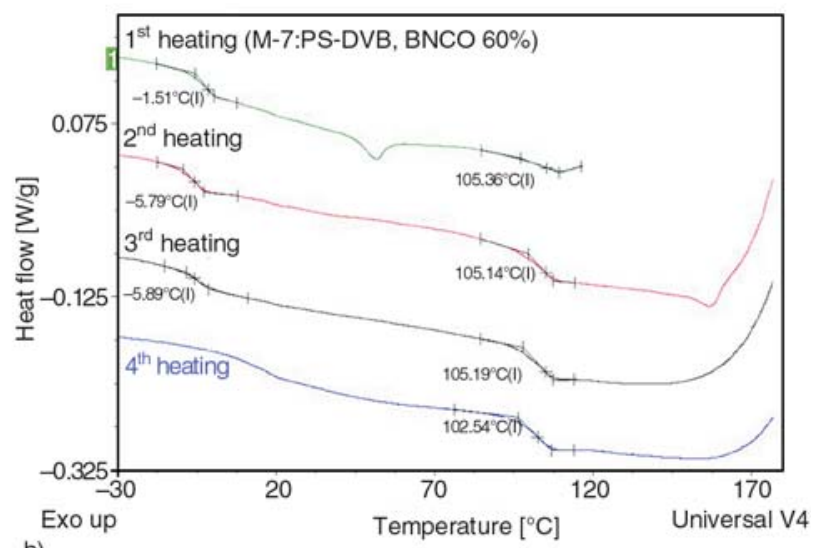

b)

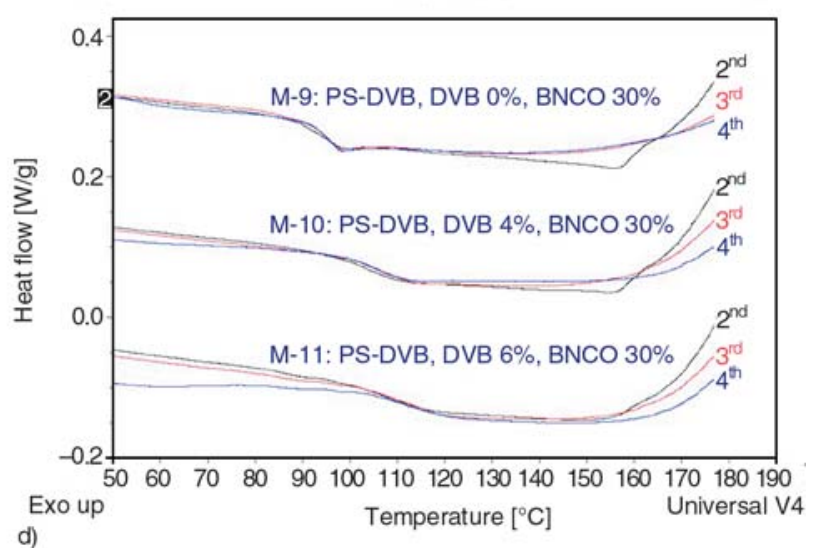

Figure 6. DSC profiles of Desmodur BL3175A and nanocapsules 
residual blocked isocyanates. Two distinct $T_{g} \mathrm{~s}$ are discernible in the DSC profile of nanocapsule M-7 (Figure 6b). The first $\mathrm{Tg}$ of approximately $-5^{\circ} \mathrm{C}$ is attributed to the incorporated $\mathrm{BNCO}$, and the second $T_{g}$ of approximately $105^{\circ} \mathrm{C}$ is attributed to the presence of partially crosslinked polystyrene. The separate and distinct $T_{g}$ s confirm distinct phase separation within nanocapsules.

The effects of time and temperature on deblocking BL3175A should also be noticed in the DSC thermograms of nanocapsules containing varying amounts of BNCO. Nanocapsules containing $>20 \%$ $\mathrm{BNCO}$ exhibited exothermic responses after the second heating cycle, while nanocapsules containing $<20 \%$ BNCO did not show similar responses (the profile is not included in Figure 6). Additionally, higher degrees of crosslinking in the shell (DVB increased from 2 to $6 \mathrm{wt} \%$ ) also required longer deblocking times (Figure 6d). Higher crosslinking of the shell is seen to promote the 'shield effect'. DSC analysis was also conducted with open samples (no lid) and closed samples (pan capped with a small hole in the center). The DSC thermograms shown in this paper were all recorded on closed pans. The thermal behaviors of both processes were similar except for the lack of exothermic responses in the open samples, which reflect the effect of crosslinking on the deblocking behavior and the reversible nature of blocked isocyanates establishing an equilibrium in sealed pans, which is consistent with the observations reported by Wicks [13].

\subsection{Hydroxyl or amine functionalized polymers encapsulated isocyanate nanocapsule}

The above synthesis technique was repeated using HEMA and TBAEMA as comonomers with styrene and divinyl benzene to encapsulate isocyanates via miniemulsion polymerization. The resulting $\mathrm{N}$-series of nanocapsules are summarized in Table 4.

\subsubsection{Encapsulation of isocyanate by hydroxyl functionalized polymer}

Isocyanate encapsulation was confirmed via FTIR characterization. The FTIR spectra of N3300A and BL3175A are re-plotted for comparison in Figures 7 and 8. The nanocapsule N-5 (Figure 7e) shows a characteristic BNCO peak at $1682 \mathrm{~cm}^{-1}$ that is not observed in the FTIR spectrum of the empty nanosphere, N-4 (Figure 7d), and confirms the presence of blocked isocyanate. However, the peak at $1725 \mathrm{~cm}^{-1}$ overlaps with the HEMA ester carbonyl of N-4.

The IR spectrum of N-5 treated with acetone to extract the encapsulated BNCO (Figure 7f) shows total disappearance of the BNCO peak at $1682 \mathrm{~cm}^{-1}$ confirming blocked isocyanate extraction from the nanocapsules by acetone. The data also suggests that a core-shell structure (isocyanate

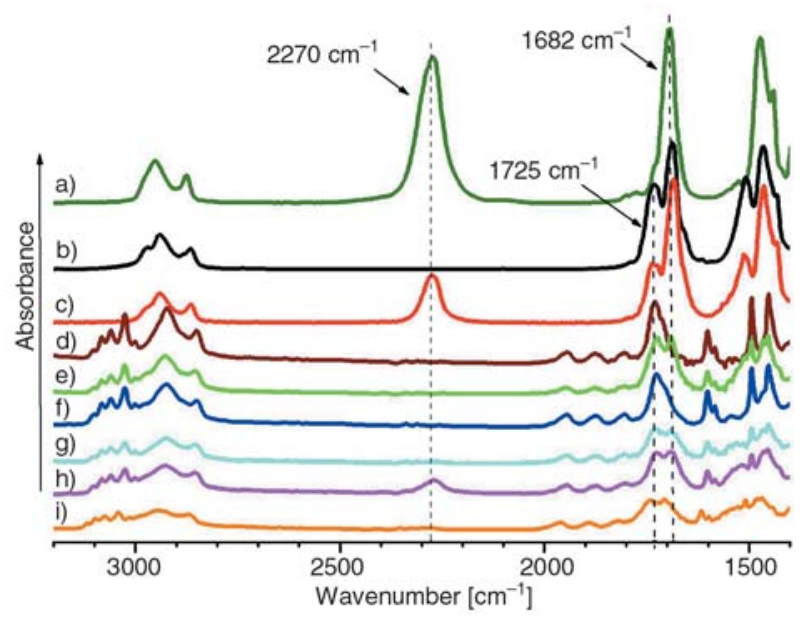

Figure 7. FTIR spectra of a) N3300A, b) BL3175A,

c) BL3175A after deblocking treatment, d) N-4, e) N-5, f) N-5 after acetone extraction, g) blend of N-4 and BL3175A after deblocking treatment, h) N-5 after deblocking treatment, and i) N-6 after deblocking treatment

Table 4. Hydroxyl or amine functionalized BNCO nanocapsules

\begin{tabular}{|c|c|c|c|c|c|}
\hline Sample & DVB* [\%] & BNCO* [\%] & $\begin{array}{c}\text { HEMA/S } \\
\text { weight ratio }\end{array}$ & $\begin{array}{c}\text { TBAEMA/S } \\
\text { weight ratio }\end{array}$ & $\begin{array}{c}\text { Particle size } \\
{[\mathbf{n m}]}\end{array}$ \\
\hline N-4 & 2 & 0 & 0.25 & - & 61 \\
\hline N-5 & 2 & 30 & 0.25 & - & 57 \\
\hline N-6 & 0 & 30 & 0.25 & - & - \\
\hline N-7 & 2 & 30 & - & 0.25 & 62 \\
\hline N-8 & 2 & 0 & - & 0.25 & - \\
\hline
\end{tabular}

*weight percentage on total monomer 
core) for nanocapsules was maintained until acetone extraction.

For isocyanate releasing studies, nanocapsules N-6 were freeze dried and treated thermally in the DSC to release the blocking agent. Figures $7 \mathrm{c}, 7 \mathrm{~g}$, and $7 \mathrm{~h}$ show the effects of deblocking treatment on BL3175A, blend of nanospheres N-4 and BL3175A, and $\mathrm{N}-5$, respectively. While the presence of the free NCO peak at $2270 \mathrm{~cm}^{-1}$ indicates deblocking of BL3175A, the peak at $1725 \mathrm{~cm}^{-1}$ supports the presence of residual BNCO. After the deblocking treatment, nanocapsule N-5 showed the characteristic NCO peak at $2270 \mathrm{~cm}^{-1}$ indicating the presence of active isocyanate functional groups. The functionalized nanocapsules are expected to have the hydroxyl groups on the outside of the shell due to their hydrophilicity. When the blend of hydroxylfunctional empty nanosphere N-4 and BL3175A was subjected to the deblocking treatment (Figure $7 \mathrm{~g}$ ), no peak was seen at $2270 \mathrm{~cm}^{-1}$ suggesting that the isocyanate generated upon deblocking had reacted with the hydroxyl groups present on the nanosphere. On the other hand, the isocyanate generated upon deblocking in nanocapsule $\mathrm{N}-5$ is retained in the core of the nanocapsule and does not react with the hydroxyl groups on the outside of the shell. The data is strong support for the efficient encapsulation of isocyanate by the hydroxyl functionalized polymer shells.

The degree of crosslinking in the shell is also demonstrated in Figure 7. Unlike nanocapsule N-5 (2\% DVB in the shell), the nanocapsule N-6 (no crosslinker in the shell) does not display the NCO peak at $2270 \mathrm{~cm}^{-1}$ after deblocking thermal treatment (Figure 7i). During deblocking, the active isocyanate flows from the uncrosslinked shell, and reacts with the hydroxyl groups on the outside of the shell. It is obvious therefore that DVB plays an important role as a crosslinker in confining the $\mathrm{BNCO}$ to the core of the nanocapsule.

\subsubsection{Encapsulation of isocyanate by amine functionalized polymer}

The nanocapsule N-7 (Figure 8e) shows a characteristic BNCO peak at $1682 \mathrm{~cm}^{-1}$ that is not observed in the FTIR spectrum of the empty nanosphere N-8 (Figure $8 d$ ). The peak at $1725 \mathrm{~cm}^{-1}$ overlaps with the TBAEMA ester carbonyl of N-8.

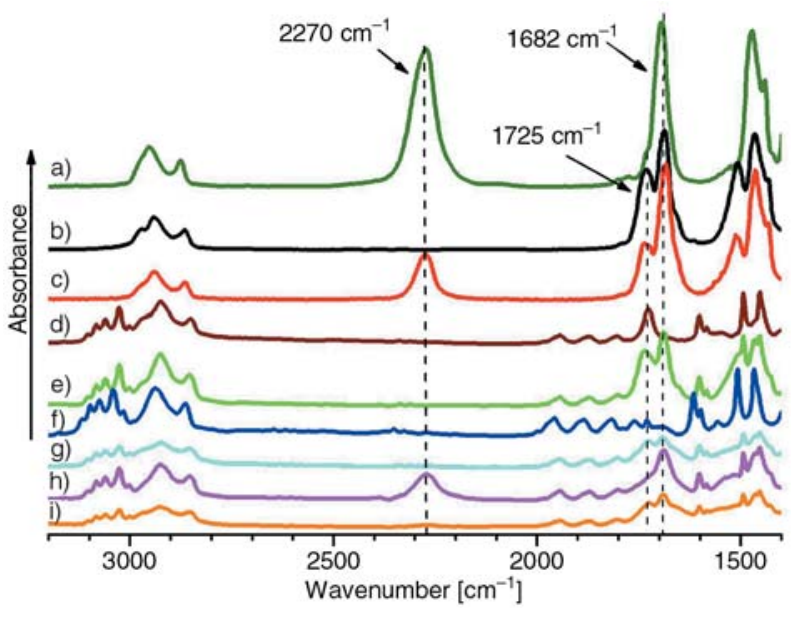

Figure 8. FTIR spectra of a) N3300A, b) BL3175A,

c) BL3175A after deblocking treatment, d) N-8, e) N-7, f) N-7 after acetone extraction, g) blend of N-8 and BL3175A after deblocking treatment, h) N-7 after deblocking treatment, and i) N-9 after deblocking treatment

The IR spectrum of N-7 treated with acetone to extract the encapsulated BNCO (Figure 8f) shows the total disappearance of the BNCO peak at $1682 \mathrm{~cm}^{-1}$ indicating that the blocked isocyanate was extracted from the nanocapsules by acetone, and supports the core (isocyanate)-shell structure of the nanocapsules. Figures $8 \mathrm{c}, 8 \mathrm{~g}$, and $8 \mathrm{~h}$ summarize the effect of deblocking treatment on BL3175A, blend of nanospheres N-8 and BL3175A, and N-7, respectively. After deblocking, nanocapsule N-7 exhibits the characteristic NCO peak at $2270 \mathrm{~cm}^{-1}$ indicating the presence of active isocyanate functional groups. This peak is not seen in the similarly treated blend of empty nanospheres $\mathrm{N}-8$ and BL3175A (Figure 8g) as the active isocyanate generated upon deblocking reacts with the TBAEMA amine groups present on the nanocapsule shell. However, the isocyanate generated upon deblocking in nanocapsule $\mathrm{N}-7$ does not react with amine groups, and remains in the core as the dissociated free isocyanate, thus confirming the isocyanate encapsulation.

The nanocapsule N-9 (Figure 8i) without DVB crosslinker in the shell does not depict a NCO peak at $2270 \mathrm{~cm}^{-1}$ after deblocking treatment. As discussed earlier, it is believed that the deblocked isocyanate is able to flow from the uncrosslinked shell and react with the amine groups on the shell periphery.

Since isocyanate deblocking appeared to be a function of time, nanocapsule N-7 was heated isother- 


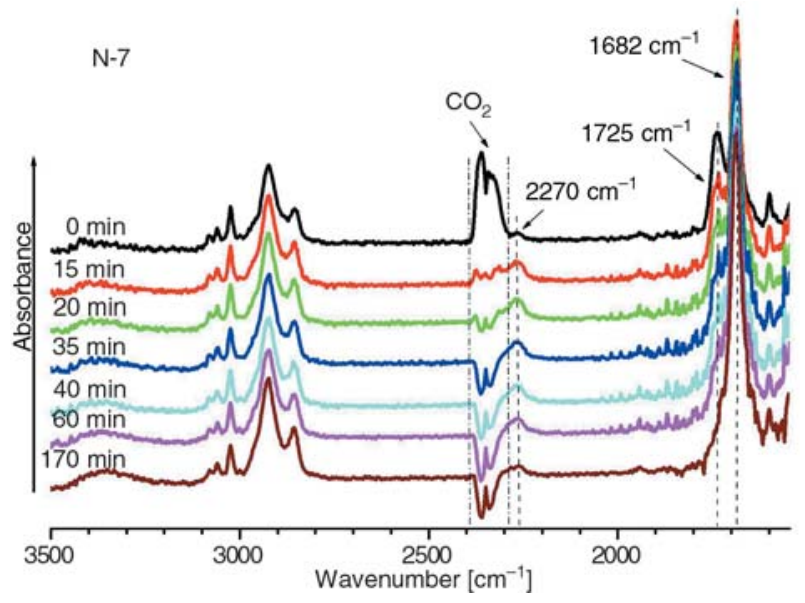

Figure 9. FTIR spectra of N-7 maintained isothermally at $140^{\circ} \mathrm{C}$

mally at $140^{\circ} \mathrm{C}$ for varying lengths of time (Figure 9). The characteristic BNCO peak at $1725 \mathrm{~cm}^{-1}$ decreased steadily with time. At 170 minutes, a small shoulder is seen that represents the TBAEMA ester carbonyl. The time-dependent deblocking behavior noted here is consistent with our earlier observations. The NCO peak at $2270 \mathrm{~cm}^{-1}$ confirms that the deblocked isocyanates were retained in the core of the nanocapsule and not consumed by the amine groups on the nanocapsule periphery.

\section{Conclusions}

Isocyanates have been successfully encapsulated into polystyrene, and hydroxyl and amine functionalized nanospheres using a commercially available blocked isocyanate. The research provides a new method for synthesizing nanocapsules containing either blocked or free isocyanates in aqueous media. The thermally dissociated isocyanate can be utilized as an active functional group in coatings and adhesive applications. Nanocapsules containing blocked isocyanates are of interest in self-healing systems where the isocyanate can be liberated by thermal treatment or extracted via solvent or reactive agents such as amines. Nanocapsule shells functionalized with amines and hydroxyls provide a ready moiety for the isocyanate to react once it is liberated from the protective shell of the nanocapsule. Quantitative characterization of the encapsulated isocyanate and the reactivity of released isocyanate are under investigation and will be reported in a subsequent article.

\section{Acknowledgements}

This material is based upon work supported by the Cooperative State Research, Education, and Extension Service, U.S. Department of Agriculture, under Agreement Nos. 2001-38202-10424 and 2006-38202-16954. The authors thank Bayer MaterialScience for donating the isocyanates used in this study.

\section{References}

[1] Arshady R.: Microspheres, microcapsules and liposomes, Volume 1. Citrus Books, London (1999).

[2] Jackson L. S., Lee K.: Microencapsulation and the food industry. Lebensmittel-Wissenschaft und -Technologie, 24, 289-297 (1991).

[3] Hildebrand G. E., Tack J. W.: Microencapsulation of peptides and proteins. International Journal of Pharmaceutics, 196, 173-176 (2000).

[4] Stenekes R. J. H., Loebis A. E., Fernandes C. M., Crommelin D. J. A., Hennink W. E.: Degradable dextran microspheres for the controlled release of liposomes. International Journal of Pharmaceutics, 214, 17-20 (2001).

[5] Landfester K.: The generation of nanoparticles in miniemulsions. Advanced Materials, 13, 765-768 (2001).

[6] Qi D-M., Bao Y-Z., Weng Z-X., Huang Z-M.: Preparation of acrylate polymer/silica nanocomposite particles with high silica encapsulation efficiency via miniemulsion polymerization. Polymer, 47, 46224629 (2006).

[7] Ni K-F., Shan G-R., Weng Z-X.: Synthesis of hybrid nanocapsules by miniemulsion (co)polymerization of styrene and $\gamma$-methacryloxypropyltrimethoxysilane. Macromolecules, 39, 2529-2535 (2006).

[8] Jang J., Bae J., Park E.: Selective fabrication of poly(3,4-ethylenedioxythiophene) nanocapsules and mesocellular foams using surfactant-mediated interfacial polymerization. Advanced Materials, 18, 354-358 (2006).

[9] Couvreur P., Barratt G., Fattal E., Legarnd P., Vauthier C.: Nanocapsule technology: A review. Critical Reviews in Therapeutic Drug Carrier Systems, 19, 99-134 (2002).

[10] Matkan J., Treleaven R. J.: Particles containing releasable fill material and method of making same. US patent 4681806, USA (1987).

[11] Irii S., Shiozaki T.: Manufacture of microcapsules. JP patent 62193641, Japan (1987).

[12] Lee W-F., Hwong G-Y.: Curing behavior of poly(vinyl chloride)-graft-2-hydroxyethyl methacrylate. European Polymer Journal, 33, 1499-1504 (1997).

[13] Wicks D. A., Wicks Z. W.: Blocked isocyanates III: Part A. Mechanisms and chemistry. Progress in Organic Coatings, 36, 148-172 (1999). 